\title{
A New Species of Rhabdias (Nematoda: Rhabditida: Rhabdiasidae) from Miyakojima Island, Okinawa, Japan
}

\author{
Naoya Sata ${ }^{1,2,4}$, Hirohiko Takeuchi ${ }^{3}$, and Takafumi Nakano ${ }^{2}$ \\ ${ }^{1}$ Meguro Parasitological Museum, Meguro-ku, Tokyo 153-0064, Japan \\ E-mail:nsata@kiseichu.org \\ ${ }^{2}$ Department of Zoology, Graduate School of Science, Kyoto University, Sakyo-ku, Kyoto 606-8502, Japan \\ E-mail:nsata@kiseichu.org \\ ${ }^{3}$ College of Bioresource Science, Nihon University, Fujisawa, Kanagawa 252-0880, Japan \\ ${ }^{4}$ Corresponding author
}

(Received 9 September 2019; Accepted 13 March 2020)

http://zoobank.org/9130D837-20DE-449A-844A-CC7F6D697444

\begin{abstract}
A new nematode species that is lung parasitic to a bufonid toad, Rhabdias kafunata sp. nov., is described and illustrated from Miyakojima island, Okinawa Prefecture, Japan. Although the new species was previously identified as R. incerta Wilkie, 1930, it is clearly distinguishable from that species by the characteristics of body proportion, buccal capsule, and esophagus. Additionally, the new species differs from other congeners inhabiting East Asia and the Russian Far East in the following features: longer body length, cup-like buccal capsule, longer esophagus length, presence of thickened anterior muscular part in esophagus, cuticular inflation less prominent in region posterior to small cephalic cuticular inflation, middle part of body and tail tip, and tail long and tapering. Partial sequences of mitochondrial cytochrome $c$ oxidase subunit I and $12 \mathrm{~S}$ rDNA genes were provided as DNA barcodes for the new species.
\end{abstract}

Key Words: Bufo gargarizans miyakonis, endoparasite, misidentification, Ryukyu Islands, nematode.

\section{Introduction}

The genus Rhabdias Stiles and Hassall, 1905 is a parasitic nematode group that dwells in the lung of amphibians and reptiles, and consists of $c a .85$ nominal species that are distributed worldwide (Kuzmin 2013). Seven species of this genus have been recorded from the Japanese and/or Ryukyu Archipelago, Japan, and two have also been identified from the adjacent continental region (Kung and Wu 1945; Wang et al. 1978; Moravec and Sey 1985; Kuzmin 2013).

Rhabdias incerta Wilkie, 1930 is a bufonid toad specific species, and was originally described from Tokyo, Japan (Wilkie 1930). This species has been recorded from the Japanese Archipelago, Miyakojima island (the sole island of the Ryukyu Archipelago where the native bufonid species is distributed) and the Russian Far East (Yamaguti 1941; Hasegawa 1984; Hasegawa and Iwatsuki 1993; Goldberg and Bursey 2002; Hasegawa and Asakawa 2004; Kuzmin 2013).

The previous studies reported the morphological differences between a western Japanese Archipelago population and the original material of $R$. incerta (Yamaguti 1941). Although morphological similarities between the Miyakojima island population and Japanese mainland population were indicated by Hasegawa (1984), the measurements of the buccal capsule of the island population, a diagnostic character of Rhabdias species (Svitin et al. 2018), were not provided at that time. Furthermore, because a recent phylogenetic study of Rhabdias suggested the occurrence of mul- tiple cryptic species-level divergences (Müller et al. 2018), the true species diversity of Rhabdias should be revealed by careful morphological observation and a molecular phylogenetic approach.

In the present study, Rhabdias specimens collected from the Miyako toad, Bufo gargarizans miyakonis Okada, 1931, obtained from Miyakojima island were inspected, and a new species is described. Additionally, mitochondrial DNA sequences of the new species are provided for future phylogenetic analyses.

\section{Materials and Methods}

Sampling and morphological examination. Specimens of Rhabdias examined in this study were obtained from six specimens of $B$. g. miyakonis which were collected from Hirara-Nishinakasone $\left(24^{\circ} 48^{\prime} 48.0^{\prime \prime} \mathrm{N}, 125^{\circ} 18^{\prime} 44.0^{\prime \prime} \mathrm{E}\right)$, Miyakojima island, Okinawa Prefecture, Japan. All captured toads were euthanized by immediate preservation at $-20^{\circ} \mathrm{C}$. The body cavity of each anuran was dissected by a longitudinal incision, and then the lungs were removed. The excised organs were dissected longitudinally, and the lumens were investigated. Most of the obtained nematode individuals were fixed with a hot $5 \%$ solution of glycerin in $70 \%$ ethyl alcohol, and the reminders were fixed with 3\% formalin and then preserved in $70 \%$ ethyl alcohol. To clear the nematode specimens, they were placed in $5 \%$ solution of glycerin in $30 \%$ ethyl alcohol, then incubated $2-3$ days at $60^{\circ} \mathrm{C}$ to 
gradually evaporate the ethyl alcohol. The cleared specimens were observed with a light microscope (OLYMPUS BX53).

The measurements were given for a holotype, followed by the range of paratypes in parentheses except for eggs. The measurements of egg were given as mean value, followed by range of holotype. All measurements were described in micrometers $(\mu \mathrm{m})$ unless otherwise stated in $\mathrm{mm}$.

Both the nematode and toad specimens examined in this study have been deposited in the Zoological Collection of Kyoto University (KUZ).

PCR and DNA sequencing. Genomic DNA of nematode specimens was extracted following the method described by Sata (2018). The partial sequence of cytochrome $c$ oxidase subunits I (COI) and $12 \mathrm{~S}$ ribosomal DNA (12S) were amplified by polymerase chain reaction (PCR) using a TaKaRa Ex Taq kit (Takara Bio, Japan) and a GeneAmp PCR Systems 2700 instrument (Applied Biosystems, USA; ABI). The primer sets used for PCR of COI was primer cocktail of NemF1_t1-NemF3_t1 and NemR1_t1-NemR3_t1, as described in Prosser et al. (2013), of 12S was 12SF and 12SR, as described in Casiraghi et al. (2004). PCR conditions were as follows: $94^{\circ} \mathrm{C}$ for $3 \mathrm{~min}$, followed by five cycles of $40 \mathrm{~s}$ at $94^{\circ} \mathrm{C}, 40 \mathrm{~s}$ at $45^{\circ} \mathrm{C}$, and $1 \mathrm{~min}$ at $72^{\circ} \mathrm{C} ; 35$ cycles of $40 \mathrm{~s}$ at $94^{\circ} \mathrm{C}, 40 \mathrm{~s}$ at $51^{\circ} \mathrm{C}$, and $1 \mathrm{~min}$ at $72^{\circ} \mathrm{C}$ and then a final extension at $72^{\circ} \mathrm{C}$ for $5 \mathrm{~min}$ for COI; $94^{\circ} \mathrm{C}$ for $3 \mathrm{~min}$, followed by 35 cycles of $45 \mathrm{~s}$ at $94^{\circ} \mathrm{C}, 45 \mathrm{~s}$ at $48^{\circ} \mathrm{C}$, and $1 \mathrm{~min}$ at $72^{\circ} \mathrm{C}$ and then a final extension at $72^{\circ} \mathrm{C}$ for $5 \mathrm{~min}$ for $12 \mathrm{~S}$. The PCR products were purified with ExoSAP-IT reagent (Affymetrix, USA). Sequencing reactions were performed using a BigDye Terminator Cycle Sequencing Kit v. 3.1 (ABI). The primer set used for cycle sequencing (CS) reaction of COI was M13F and M13R, as described in Messing (1983); and the primer set for CS reaction of $12 \mathrm{~S}$ corresponded to that used for PCR reaction. The products were cleaned by ethanol precipitation and sequenced with an ABI 3130xl Genetic Analyzer. In total, four DNA sequences from the present specimens of Rhabdias were determined, and deposited with the International Nucleotide Sequence Database Collaboration (INSDC) through the DNA Data Bank of Japan.

\section{Results}

\section{Genus Rhabdias Stiles and Hassall, 1905 Rhabdias kafunata sp. nov.} (Fig. 1)

Rhabdias incerta non Wilkie, 1930: Hasegawa 1984: 16, figs 11-13; Kuzmin 2013: 41, 44 (in part), fig. 21, table 9.

Diagnosis. The East Asian Rhabdias, with long and slender body. Buccal capsule small and cup-like. Esophagus 694-878 long, anterior muscular region thickened. Cuticular inflation less prominent in region posterior to small cephalic cuticular inflation, middle part of body and tail tip. Tail long and tapering sensu Wilkie (1930) and Yuen (1965).

Material examined. Holotype: KUZ Z2572, whole specimen, adult female, obtained from the lung of a specimen of
Bufo gargarizans miyakonis (KUZ R77141), collected from Hirara-Nishinakasone $\left(24^{\circ} 48^{\prime} 48.0^{\prime \prime} \mathrm{N}, 125^{\circ} 18^{\prime} 44.0^{\prime \prime} \mathrm{E}\right)$, Miyakojima island, Okinawa Prefecture, Japan on 14 November 2016. Paratypes: KUZ Z2573-Z2579, whole specimens obtained from the lung of the same specimen of holotype's host and other B. g. miyakonis specimens (KUZ R77142, R77143, R77147, R77149), collected from the same locality and data as the holotype's host-specimen; KUZ Z2581, one prepared slide of a section of anterior end of a female; and KUZ Z2580, remaining body specimen of KUZ Z2581. KUZ Z2581 and Z2580 were obtained from the lung of a B. g. miyakonis specimen (KUZ R77148), collected from the same locality and data as the holotype's host-specimen.

Additional materials: in total, 164 adult and young females were obtained from the lung of B. g. miyakonis; KUZ Z2702 and Z2703, 57 whole specimens, and six non-deposited specimens for the present and future molecular studies, obtained from KUZ R77149; KUZ Z2704 and Z2705, eight whole specimens, and one non-deposited specimen for a future molecular study, from KUZ R77147; KUZ Z2706, five whole specimens, and three non-deposited specimens for a future molecular study, from KUZ R77143; KUZ Z2707 and Z2708, 33 whole specimens from KUZ R77142; KUZ Z2709 and Z2710, 44 whole specimens, and five non-deposited specimens for the present and future molecular studies, from KUZ R77141; and KUZ Z2711, two whole specimens, from KUZ R77148.

Type locality. Hirara-Nishinakasone $\left(24^{\circ} 48^{\prime} 48.0^{\prime \prime} \mathrm{N}\right.$, $\left.125^{\circ} 18^{\prime} 44.0^{\prime \prime} \mathrm{E}\right)$, Miyakojima island, Okinawa Prefecture, Japan.

Type host. Bufo gargarizans miyakonis Okada, 1931 (Amphibia: Bufonidae); site of infection: lung. A total of 173 individuals were obtained from six specimens of B. g. miyakonis; mean intensity (mean \pm standard error, followed by ranges): $28.8 \pm 10.5,3-66$.

Description. General. Body long and slender with tapered extremities, body becoming wider at region between anterior end of genital system and vulva. Cephalic end with six lips, each lip with a minute papilla. Amphids situated on posterior surface of lateral lips. Oral opening rounded-hexagonal. Small cuticular inflation present on cephalic region. Cuticular inflation less prominent in region posterior to small cephalic cuticular inflation, middle part of body and tail tip.

Body length 11.06 (10.99-16.77) $\mathrm{mm}$, and maximum width 236 (204-355). Body length/maximum body width $=46.9(44.8-53.9)$. Body width at junction of esophagus and intestine 157 (141-239), at vagina 181 (181-340), and at anus 98 (91-144). Vestibulum short, 4.3 (2.5-6) $(n=7)$. Buccal capsule small, cup-like in lateral view, 16 (15$19)$ deep $(n=7), 26(21-30)$ wide $(n=7)$. Anterior muscular region of esophagus thickened. Length of esophagus 758 (694-878) long [6.9\% (4.2-7.3) of body length] with width of $43(33-46)$ at anterior end, $56(47-59)$ at thickened muscular part, 39 (39-56) at nerve ring, $80(80-101)$ at bulbar part. Nerve ring and excretory pore $227(193-231)$ [2.1\% (1.2-2.1\%) of body length] and 262 (228-277) [2.4\% (1.5$2.5 \%$ ) of body length], respectively, from cephalic end, and 

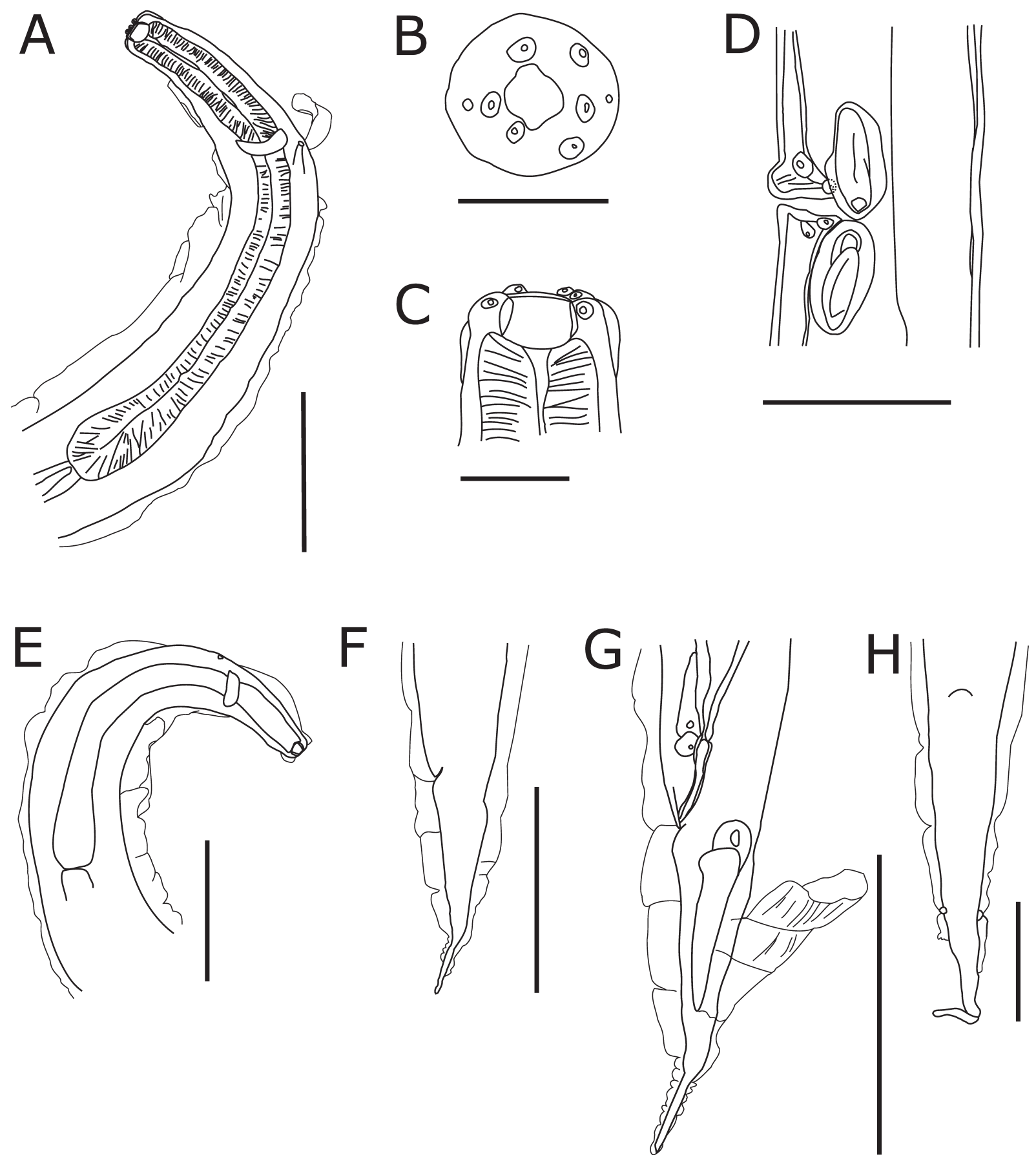

Fig. 1. Rhabdias kafunata sp. nov., holotype (KUZ Z2572: A, C, D, H), paratypes (KUZ Z2581: B; KUZ Z2578: E-G). A, anterior region, lateral view; B, cephalic region, apical view; $\mathrm{C}$, cephalic region, lateral view; $\mathrm{D}$, vulvar region, lateral view; E, cuticular inflation in anterior region, lateral view; F, cuticular inflation in caudal region; G, caudal region, lateral view; H, phasmids, ventral view. Scale bars: $200 \mu \mathrm{m}$ (A), $40 \mu \mathrm{m}(\mathrm{B}, \mathrm{C}), 180 \mu \mathrm{m}(\mathrm{D}), 400 \mu \mathrm{m}(\mathrm{E}), 450 \mu \mathrm{m}(\mathrm{F}, \mathrm{G})$, and $150 \mu \mathrm{m}(\mathrm{H})$.

208 (166-208) [27.4\% (20.8-27.4\%) of esophagus length] and 247 (195-259) [32.6\% (26.2-32.6\%) of body length], respectively, from anterior end of esophagus. Intestine thick walled. Rectum short, with thin wall. Vulva 6.18 (5.97-8.32) $\mathrm{mm}$ from cephalic end, and located at middle to posterior of body [55.9\% (49.6-55.9\%) of body length], having slightly salient lips. Genital system amphidelphic, anterior limb turned posteriorly at $2.18(2.18-3.28) \mathrm{mm}$ from cephalic end, and posterior limb turned anteriorly at 2.48 (1.45-3.25) $\mathrm{mm}$ from tail end. Uteri long, tubular, filled with numerous eggs. Tail long, gradually tapering from anus, and 477 (376$493)$ long [4.3\% (2.8-4.3\%) of body length]. Most specimens possessing few numbers of lateral pores. Phasmids located both lateroventral sides, $166(144-200)$ from tail end $(n=4)$. Eggs elliptical, $101(89-107)$ by $47(42-55)(n=10)$, thin shelled, most eggs containing first stage larva.

Etymology. The specific name kafunata is a noun from the word locally used in the Miyako Islands for B. g. miyakonis, the host toad of the new species (Okinawa Center of Language Study 1999-2003); thus not a Latin or Latinized 
word.

DNA Sequences. In total, 4 sequences were determined: one specimen obtained from a same host toad as holotype (KUZ R77141), 2 sequences-COI (LC496790; 655 bp), and 12S (LC496792; $475 \mathrm{bp}$ ); one specimen obtained from a same host toad as paratypes (KUZ R77148), 2 sequencesCOI (LC496791; 655 bp), and 12S (LC496793; 475 bp).

Remarks. Rhabdias kafunata sp. nov. can be discriminated from the other nine congeners recorded from East Asia and the Russian Far East as follows. The new species, of which body length ranges from 10.99 to $16.77 \mathrm{~mm}$, can be distinguished from the following four Rhabdias species: $R$. globocephala Kung and Wu, 1945 [3.18-6.88 mm in Kuzmin (2005)], R. montana Yamaguti, 1954 (6.0-7.8 mm; Yamaguti 1954), R. nipponica Yamaguti, 1935 (4.18-5.04 mm; Yamaguti 1935), and R. polypedatis Yamaguti, 1941 (4.2-5.2 mm; Yamaguti 1941). Rhabdias kafunata sp. nov. can be discriminated from R. bermani Rausch, Rausch, and Atrashkevich, 1984 and $R$. rhacophori Yamaguti, 1941 by their esophagus lengths, because latter two species possess much shorter esophagus than that of the new species (Yamaguti 1941; Rausch et al. 1984). Rhabdias kafunata sp. nov. also differs from $R$. bermani in its possession of the thickened anterior muscular part in esophagus. Rhabdias kafunata sp. nov. is distinguishable from $R$. japalurae Kuzmin, 2003, and $R$. tokyoensis Wilkie, 1930 by having a long and tapering tail. Unlike the new species, $R$. japalurae and $R$. tokyoensis have short and conical tails. Rhabdias kafunata sp. nov. also differs from $R$. japalurae in the absence of a cuticular inflation at the region middle of esophagus. The new species possesses a cup-like buccal capsule; this characteristic can discriminate $R$. kafunata sp. nov. from R. bicornis Lu, 1934, which has a funnel-like buccal capsule ( $\mathrm{Lu} \mathrm{1934).}$

\section{Discussion}

As previously identified as Rhabdias incerta (Hasegawa 1984; Kuzmin 2013), R. kafunata sp. nov. morphologically resembles the Rhabdias species described by Wilkie (1930). However, the new species can be clearly discriminated from $R$. incerta sensu Wilkie (1930) by its comparatively slender body, smaller buccal capsule, and shorter esophagus. The new species is also distinguishable from the western Japanese-mainland population of $R$. incerta defined by Yamaguti (1941) by having comparatively slender body, and shorter esophagus. Furthermore, R. kafunata sp. nov. differs from the Russian Far East R. incerta (Kuzmin 2013) by tis narrower width of buccal capsule and shorter esophagus. Although some key characters were not provided, the most numerical characteristics of the Miyakojima island population of " $R$. incerta" reported by the previous studies (Hasegawa 1984; Kuzmin 2013) are concordant with those of the new species. Thus, the nematodes of Rhabdias from Miyakojima island in Hasegawa (1984) and Kuzmin (2013) might belong to R. kafunata sp. nov. described in the present study.

Considering the high host specificity of $R$. incerta to na- tive bufonid toads in Japan (Wilkie 1930; Yamaguti 1941; Goldberg and Bursey 2002; Hasegawa and Asakawa 2004), their inter-population gene flow may largely depend on the dispersal range of its bufonid hosts. The deep genetic divergence between two subspecies of Bufo japonicus Temminck and Schlegel, 1838, which are parapatrically distributed in Honshu, Japan, was revealed by phylogenetic studies (Kawamura et al. 1990; Igawa et al. 2006). Therefore, morphological differences between eastern and western Japanese-mainland populations of $R$. incerta that were reported by Yamaguti (1941) may reflect genetic divergences between those two populations. Because Russian Far East nematodes identified as $R$. incerta were found from the bufonid Pseudepidalea raddei (Strauch, 1876) (Kuzmin 2013), it is also highly possible that the Russian population might have genetically diverged from the Japanese mainland populations. The Russian Far East R. incerta in Kuzmin (2013) differs from the Wilkie's $R$. incerta in having a shallower buccal capsule. However, the measurements provided in the previous studies cannot discriminate the Russian Far East population from the western Japanese-mainland population (Yamaguti 1941; Kuzmin 2013). Accordingly, the taxonomic status of western Japanese-mainland and Russian Far East populations, which have been traditionally deemed $R$. incerta, should be clarified by further morphological investigation and molecular phylogenetic analysis.

\section{Acknowledgments}

We are most grateful to Dr. Takashi Haramura (Rakuno Gakuen University) for his support for the field work in the Miyakojima, and two anonymous reviewers and Dr. Daisuke Uyeno (Kagoshima University) for their constructive comments and suggestions on the manuscript. Collection of the host specimens in Miyakojima island was carried out with the permission of Miyakojima City (Approval Number: 289). We also thank R. J. Frampton from Edanz Group (www.edanzediting.com/ac) for editing a draft of this manuscript. This study was financially supported by JSPS KAKENHI Grant number JP17K20064.

\section{References}

Casiraghi, M., Bain, O., Guerrero, R., Martin, C., Pocacqua, V., Gardner, S. L., Franceschi, A., and Bandi, C. 2004. Mapping the presence of Wolbachia pipientis on the phylogeny of filarial nematodes: evidence for symbiont loss during evolution. International Journal for Parasitology 34: 191-203.

Goldberg, S. R. and Bursey, C. R. 2002. Helminth of 10 species of anurans from Honshu Island, Japan. Comparative Parasitology 69: $162-176$.

Hasegawa, H. 1984. Helminth fauna of five Okinawan amphibian species. The Biological Magazine Okinawa 22: 11-22. [In Japanese with English abstract]

Hasegawa, H. and Asakawa, M. 2004. Parasitic nematodes recorded from amphibians and reptiles in Japan. Current Herpetology 23: 27-35. 
Hasegawa, H. and Iwatsuki, N. 1993. Prevalence of helminths in Bufo gargarizans miyakonis on Miyakojima Island, Okinawa, Japan. Akamata 8: 16-18. [In Japanese]

Igawa, T., Kurabayashi, A., Nishioka, M., and Sumida, M. 2006. Molecular phylogenetic relationship of toads distributed in the Far East and Europe inferred from the nucleotide sequences of mitochondrial DNA genes. Molecular Phylogenetics and Evolution 38: 250-260.

Kawamura, T., Nishioka, M., Sumida, M., and Ryuzaki, M. 1990. An electrophoretic study of genetic differentiation in 40 populations of Bufo japonicus distributed in Japan. Scientific Report of the Laboratory for Amphibian Biology, Hiroshima University 10: $1-51$.

Kung, C. C. and Wu, H. W. 1945. Parasitic nematodes of amphibians from Pehpei, Szechwan, China. Sinensia 16: 73-83.

Kuzmin, Y. I. 2005. The description of Rhabdias globocephala (Nematoda, Rhabdiasidae) from the new host Buergeria pollicaris (Amphibia, Rhacophoridae). Vestnik Zoologii 39: 9-14.

Kuzmin, Y. [I.] 2013. Review of Rhabdiasidae (Nematoda) from the Holarctic. Zootaxa 3639: 1-76.

Lu, S. C. 1934. On Rhabdias, a genus of parasitic Nematoda, of Nanking. Sinensia 5: 164-172.

Messing, J. 1983. New M13 vectors for cloning. Pp. 20-78. In: Wu, R., Grossman, L., and Moldave, K. (Eds) Methods in Enzymology Recombinant DNA, Part C. Vol. 101. Academic Press, New York.

Moravec, F. and Sey, O. 1985. Some nematode parasites of frogs (Rana spp.) from North Viet Nam. Parasitologia Hungarica 18: 63-77.

Müller, M. I., Morais, D. H., Costa-Silva, G. J., Aguiar, A., Ávila, R. W., and da Silva, R. J. 2018. Diversity in the genus Rhabdias (Nematoda, Rhabdiasidae): Evidence for cryptic speciation. Zoologica Scripta 47: 595-607.

Okinawa Center of Language Study. 1999-2003. Miyako Dialect Dictionary. Available at http://rlang.lib.u-ryukyu.ac.jp/rlang/myk/ index.html (31 July 2019).

Prosser, S. W. J., Velarde-Aguilar, M. G., León-Règagnon, V., and Hebert, P. D. N. 2013. Advancing nematode barcoding: a primer cocktail for the cytochrome $c$ oxidase subunit I gene from vertebrate parasitic nematodes. Molecular Ecology Resources 13: 1108-1115.

Rausch, R. L., Rausch, V. R., and Atrashkevich, G. I. 1984. Rhabdias bermani sp. n. (Nematoda, Rhabdiasidae) from a salamander Hynobius keyserlingi in the Soviet Far East. Zoologicheskii Zhurnal 63: 1297-1304. [In Russian with English abstract]

Sata, N. 2018. Allopatric speciation of Meteterakis (Heterakoidea: Heterakidae), a highly dispersible parasitic nematode, in the East Asian islands. Parasitology International 67: 493-500.

Stiles, C. W. and Hassall, A. 1905. U. S. Department of Agriculture, Bureau of Animal Industry-Bulletin No. 79. The Determination of Generic Types. And a List of Roundworm Genera, With Their Original and Type Species. Government Printing Office, Washington, $150 \mathrm{pp}$.

Svitin, R., Kuzmin, Y. [I.], and du Preez, L. 2018. Molecular and morphological characterisation of Rhabdias picardiae Junker, Lhermitte-Vallarino et Bain, 2010 (Nematoda: Rhabdiasidae) from Delaland's river frog, Amietia delalandii (Duméril et Bibron, 1841) (Amphibian: Pyxicephalidae) in South Africa. Acta Parasitologica 63: 55-64

Wang, P. Q., Zhao, Y., and Chen, C. 1978. On some nematodes from vertebrates in South China. Journal of Fujina Normal University, Natural Science 2: 75-90. [In Chinese]

Wilkie, J. S. 1930. Some parasitic nematodes from Japanese Amphibia. Annals and Magazine of Natural History, Tenth Series 6: 606-614.

Yamaguti, S. 1935. Studies on the helminth fauna of Japan. Part 10. Amphibian nematodes. Japanese Journal of Zoology 6: 387-392.

Yamaguti, S. 1941. Studies on the helminth fauna of Japan. Part 34. Amphibian nematodes, II. Japanese Journal of Zoology 9: 397-408, $1 \mathrm{pl}$.

Yamaguti, S. 1954. Helminth fauna of Mt. Ontake. Part 1. Nematoda and Acanthocephala. Acta Medica Okayama 8: 386-392.

Yuen, P. H. 1965. Some studies on the taxonomy and development of some rhabdisoid and cosmocercoid nematodes from Malayan amphibians. Zoologischer Anzeiger 174: 275-298. 